\title{
Validación del cuestionario reducido de afrontamiento al dolor crónico en la población cienfueguera con dolor crónico de espalda [2017-2018]
}

Validation of the coping strategies to chronic pain reduced questionnaire in the cienfuegan population with chronic back pain (2017-2018)
A. J. Pomares Avalos¹, D. F. Zaldívar Pérez², R. López Fernández³ y E. J. Bernal Valladares

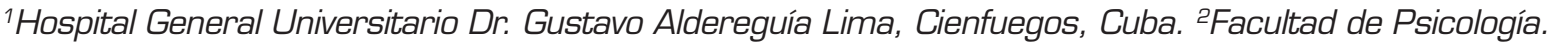 Universidad de La Habana, Cuba. 'Universidad Metropolitana de Ecuador, Universidad Carlos Rafael Rodríguez, Cienfuegos, Cuba

\section{ABSTRACT}

General objective: To determine the validity and reliability of the Coping Strategies to Back Pain Reduced Questionnaire in the Cienfuegan population with chronic back pain.

Material and method: The investigation consisted in the development of a technology to validate this Coping Strategies to Back Pain Reduced Questionnaire. The analysis of the validity of content was done through the experts's opinion and the constructo validity, starting from the factorial analysis. The analysis of the reliability was carried out through the internal consistency, calculating the Alpha coefficient of Cronbach and the temporary stability through the test retest. As part of the process of validation it was carried out a linguistic and cultural adaptation of the instrument. The study was done in the General University Hospital "Dr. Gustavo Aldereguía Lima", Cienfuegos province, Cuba, from September, 2017 to October 2018. A systematic aleatory sampling was used, being conformed the sample by 162 patients. The statistical analysis of the information was carried out in the Statistical Package SPSS.

Results: The experts considered the modification of items $2,3,6,8,10,15,16$ and 22 for a better understanding. This analysis allowed to identify the articles that

\section{RESUMEN}

Objetivo general: Determinar la validez y fiabilidad del Cuestionario Reducido de Afrontamiento al Dolor Crónico en la población cienfueguera con dolor crónico de espalda.

Material y método: La investigación consistió en el desarrollo de una tecnología para validar el Cuestionario Reducido de Afrontamiento al Dolor Crónico. El análisis de la Validez de Contenido se llevó a cabo a través del juicio de expertos y la validez de Constructo a partir del Análisis Factorial. El análisis de la Fiabilidad se realizó a través de la Consistencia Interna, calculando el coeficiente Alfa de Cronbach y la Estabilidad Temporal a través del Test Retest. Como parte del proceso de Validación se llevó a cabo la adaptación lingüística y cultural del instrumento. El estudio se realizó en el Hospital "Dr. Gustavo Aldereguía Lima" de la provincia de Cienfuegos, Cuba, de septiembre de 2017 a octubre de 2018. Se utilizó un muestreo aleatorio sistemático, quedando conformada la muestra por 162 pacientes. El análisis estadístico de la información se realizó en el paquete estadístico SPSS.

Resultados: Los expertos consideraron la modificación de los ítems 2, 3, 6, 8, 10, 15, 16 y 22 para una mejor comprensión. Este análisis permitió identificar los ítems que podían resultar confusos dentro del instrumento. Mediante el análisis factorial se identificó
Pomares Avalos AJ, Zaldívar Pérez DF, López Fernández R, Bernal Valladares EJ. Validación del cuestionario reducido de afrontamiento al dolor crónico en la población cienfueguera con dolor crónico de espalda (2017-2018). Rev Soc Esp Dolor 2019;26(5):276-283
Recibido: 19-03-2019

Aceptado: 08-07-2019

Correspondencia: Ahmed José Pomares Avalos ahmed.pomares@gal.sld.cu 
could be confused inside the instrument. Through factorial analysis the presence of six correlated factors were identified, which represented $71 \%$ of the accumulated. It was obtained a $\alpha$ of global Cronbach 0,853 and the temporary stability of the instrument was demonstrated.

Conclusion: The results confirm the validity and dependability of the Coping Strategies to the Chronic Pain Reduced Questionnaire in the Cienfuegan population with chronic back pain.

Key words: Coping strategies, chronic pain, back pain, validation, questionnaire. la presencia de seis factores correlacionados entre sí, que representó el $71 \%$ del acumulado. Se obtuvo un $\alpha$ de Cronbach global de 0,853 y quedó demostrada la estabilidad temporal del instrumento.

Conclusión: Los resultados confirman la validez y confiabilidad del Cuestionario Reducido de Afrontamiento al Dolor Crónico en la población cienfueguera con dolor crónico de la espalda.

Palabras clave: Afrontamiento, dolor crónico, dolor de espalda, validación, cuestionario.

\section{INTRODUCCIÓN}

A partir de los trabajos de Melzack y Wall, el dolor ha sido concebido multidimensionalmente, donde interaccionan factores biológicos, psicológicos y sociales (1).

Dentro de los factores psicológicos, el afrontamiento se ha convertido en uno de los temas que más importancia ha cobrado en el abordaje del paciente con dolor crónico. Se ha considerado que el dolor crónico requiere, como fuente de estrés, un esfuerzo adaptativo facilitado por las estrategias de afrontamiento que el individuo aporta a dicha situación (2-4).

Diversos instrumentos se han diseñados y validados con el objetivo de evaluar las estrategias de afrontamiento en los pacientes con dolor crónico $[5,6]$.

El Cuestionario de Afrontamiento al Dolor Crónico (CAD) de los autores Soriano y Monsalve, que proviene del ámbito cultural español, persigue estos objetivos. Redactado con un lenguaje claro y sencillo, no evidencia limitaciones culturales o lingüísticas con respecto a su uso en países de habla hispana. Compuesto por 31 ítems, recoge 6 factores considerando como estrategias activas (distracción, autocontrol mental, autoafirmación y búsqueda de información] y estrategias pasivas (religión y catarsis] [7].

Sin embargo, a referencia de los propios autores, pese a que la versión original resultó útil y fiable, las características de los pacientes con dolor crónico, llevaron a reducir el número de ítems a emplear. Esta situación generó que se elaborara una versión reducida de la prueba, finalmente conformada por 24 ítems, que agrupó los mismos factores iniciales (CAD-R) (8).

En Cuba, pese a no existir registros estadísticos de los pacientes con dolor crónico de la espalda, ha quedado demostrado que es un importante problema de salud pública [9].

La provincia de Cienfuegos tiene un comportamiento similar al resto del país, sin embargo no cuenta con instrumentos estandarizados y adaptados al contexto que permitan evaluar las estrategias de afrontamiento en los pacientes con dolor crónico de la espalda (10).

Surge así la siguiente investigación que tiene como objetivo determinar la validez y fiabilidad del Cuestionario Reducido de Afrontamiento al Dolor Crónico en la población cienfueguera con dolor crónico de la espalda.

\section{MATERIAL Y MÉTODO}

La investigación consistió en el desarrollo de una tecnología para validar el Cuestionario Reducido de Afrontamiento al Dolor Crónico mediante el análisis de la Validez de Contenido y de Constructo y el análisis de la Fiabilidad a través de la Consistencia Interna y la Estabilidad Temporal. Como parte del proceso de validación se realizó una adaptación lingüística y cultural del instrumento.

El estudio se llevó a cabo en el Hospital "Dr. Gustavo Aldereguía Lima" de la provincia de Cienfuegos, en el periodo comprendido de septiembre de 2017 a octubre de 2018. Se realizó un muestreo aleatorio sistemático, quedando conformada la muestra por 162 pacientes que cumplieron los siguientes criterios de inclusión: pacientes que pueden estar en condiciones de hospitalización o ambulatorios, que han sido valorados inicialmente por especialistas de Ortopedia y Medicina Física y Rehabilitación, con diagnóstico de dolor crónico de la espalda, con 6 meses de evolución del dolor y que dieron su consentimiento para participar en el estudio.

Para el proceso de validación se conformaron dos grupos diferentes, uno encargado de la adaptación lingưística y cultural del instrumento y otro en calidad de jueces expertos que llevaron cabo la validación de contenido.

\section{Adaptación lingüística y cultural}

En un primer momento el Cuestionario Reducido de Afrontamiento al Dolor Crónico fue sometido a un proceso de adaptación lingüística y cultural, con el objetivo de aportar mayor solidez al estudio y evitar cualquier sesgo en la información. Se solicitó la ayuda a un grupo de cinco psicólogos y un especialista en gramática, redacción y estilo de la lengua española, que llevaron a cabo la revisión del componente lingüístico, desde una perspectiva del contexto en que se aplicaría el instrumento.

Se empleó como método el Análisis Lingüístico de ítems, que consiste en la revisión semántica del instrumento con el objetivo de identificar términos de poco uso en el contexto donde se pretende aplicar o términos donde el significado sea diferente a lo aceptado en este, que permita su sustitución por otro de mejor comprensión. 


\section{Análisis de la validez y fiabilidad}

La validez de contenido se efectuó a través del criterio de expertos (11). Para la selección de los jueces expertos se utilizó el cálculo del coeficiente de competencia $(K)(12)$.

Inicialmente se solicitó la ayuda de 12 posibles potenciados, de los cuales 10 obtuvieron valores por encima de 0,80 de coeficiente de competencia, clasificando como expertos.

Los jueces expertos se caracterizan por ser licenciados en Psicología de diferentes provincias del país, ocho doctores en Ciencias Psicológicas, dos doctores en Ciencias de la Salud. Tienen como promedio alrededor de 32 años de experiencia asistencial, docente e investigadora, lo cual brinda mayor solidez a sus criterios. El número de publicaciones y la participación en eventos nacionales e internacionales es elevado.

A los expertos identificados se les solicitó que emitieran su valoración en cuanto a los criterios de Validez propuestos por Escobar-Pérez y Cuervo-Martínez (11). Estos autores proponen cuatro criterios básicos:

- Claridad: el ítem se comprende fácilmente.

- Coherencia: el ítem guarda relación con lo que se pretende evaluar.

- Relevancia: el ítem es esencial para evaluar lo que se pretende.

- Suficiencia: los ítems propuestos son suficientes para evaluar en su totalidad lo que se pretende.

A los jueces expertos se les entregó una guía de observación del instrumento que se utilizó para la recogida de la información. Se estableció una escala numérica para cada ítem en cada una de las categorías propuestas por sus autores: claridad, coherencia, relevancia y suficiencia, donde 1 expresa la menor expresión del indicador y 3 la mejor.

Para el análisis se consideró que el contenido del ítem era adecuado en el instrumento si para todos los criterios evaluados obtenía como mínimo el $70 \%$ de las respuestas emitidas en la categoría 3. En caso contrario se reevaluó el ítems.

La validez de constructo se realizó a través del análisis factorial, por componentes principales. Se comprobó el ajuste del modelo a partir de la prueba de esfericidad de Bartlett y se adoptó como criterio para considerar ajuste la $\mathrm{p} \leq 0,05$.

Posteriormente, se realizó la elección de los componentes a retener, siguiendo el criterio de normalización de Kaiser y la rotación utilizada es Varimax. Para el análisis de los factores se consideró la correlación ítemfactor en la matriz rotada, cuyo punto de corte para considerar una correlación significativa fue 0,5.

El análisis de la fiabilidad incluyó la estabilidad y la consistencia interna. Para evaluar la consistencia interna se aplicó el coeficiente Alfa de Cronbach global, eliminando un ítem sucesivamente y por factores.

En el caso de la estabilidad temporal se aplicó el instrumento en dos ocasiones sucesivas a un mismo grupo de sujetos con un intervalo de 15 días, tiempo que se consideró suficiente para que no recordaran las respuestas de la primera aplicación y, a la vez, no existieran cambios en el objeto de evaluación. Para comprobar dicha estabilidad en el tiempo se aplicó el coeficiente de correlación de Pearson.

\section{Aspectos éticos}

Para la investigación se tuvo en cuenta los aspectos éticos y jurídicos en la obtención de la información. Se utilizó el consentimiento informado de los pacientes y de la institución para la realización de la misma.

\section{RESULTADOS}

La muestra la componen 162 pacientes con dolor crónico de la espalda; de ellos, el 38,9\% eran hombres y el 61,1\% eran mujeres. El 22,2\% de los pacientes se encuentran en edades comprendidas entre los 20 y los 40 años; el 51,9\% entre 41 y 60 años y el $25,9 \%$ más de 60 años. El 51,9\% tiene más de grado 12 de escolaridad y el $48,1 \%$ menos de grado 12 de escolaridad. El 22,2 \% son jubilados o amas de casa, el 5,6\% son trabajadores que realizan esfuerzo físico y el 70,4 \% son trabajadores que no realizan esfuerzo físico.

\section{Adaptación lingüística y cultural}

El grupo encargado de la revisión lingüística y cultural del instrumento refirió que el mismo estaba redactado con frases propias del contexto social cubano. No se identificaron palabras o términos que fuera necesario modificarlas o cambiarlas por no ajustarse desde el punto de vista cultural a la población cienfueguera.

\section{Validez de contenido}

Al realizarse el análisis de contenido, en cuanto al criterio de claridad del instrumento, los jueces expertos recomendaron una serie de modificaciones a los ítems $2,3,6,8,10,15,16$ y 22 , con el objetivo de que estos reactivos fueran mejor comprendidos por parte de los pacientes y así lograr el objetivo del cuestionario. La Tabla I muestra las modificaciones propuestas por el grupo de expertos y la reestructuración de los mismos.

Estas sugerencias se devolvieron a los expertos para que emitieran un juicio de valor. La totalidad de los expertos dio una valoración de 3, por lo que consideraron adecuadas las propuestas dadas.

En relación con los criterios de coherencia y relevancia, todos los expertos coincidieron en que los ítems guardan relación y son esenciales para el logro de lo que se pretende evaluar: el modo de afrontamiento. Con respecto a la categoría suficiencia, la totalidad de los expertos marcaron la opción 3, considerando de esta forma que el número de ítems es suficiente para alcanzar el objetivo del instrumento.

\section{Validez de constructo: análisis factorial por componentes principales}

Las Tabla II y III muestran los resultados obtenidos en el Análisis Factorial. Se obtuvo un índice KMO de 0,639 claramente satisfactorio. En el test de esfericidad de Bartlett se obtuvo un estadígrafo Chi cuadrado de 2830.637, con 276 de libertad y una probabilidad 
TABLA I

MODIFICACIÓN A LOS ÍTEMS A PARTIR DEL CRITERIO DE EXPERTOS

\begin{tabular}{|c|c|c|}
\hline Ítem & CAD-R. Soriano y Monsalve & Expertos en Cuba \\
\hline 2 & $\begin{array}{l}\text { Hablo con un profesional (médico, psicólogo, } \\
\text { sacerdote, etc.) del problema para que me } \\
\text { ayude a hacerle frente }\end{array}$ & $\begin{array}{l}\text { Cuando tengo dolor hablo con un profesional para } \\
\text { que me ayude }\end{array}$ \\
\hline 3 & Rezo para curarme & Rezo para curar mi dolor \\
\hline 6 & Cuando tengo dolor no me rindo, peleo & Cuando tengo dolor lucho para vencerlo \\
\hline 8 & $\begin{array}{l}\text { Hablo con alguien que puede hacer algo } \\
\text { concreto sobre mi dolor }\end{array}$ & $\begin{array}{l}\text { Hablo con alguien que pueda ayudarme a hacer } \\
\text { frente a mi dolor }\end{array}$ \\
\hline 10 & $\begin{array}{l}\text { Cuando tengo dolor les digo a los demás lo } \\
\text { mucho que me duele, pues el compartir mis } \\
\text { sentimientos me hace encontrarme mejor }\end{array}$ & $\begin{array}{l}\text { Digo a los demás lo mucho que me duele, pues el } \\
\text { compartir mis sentimientos me hace sentir mejor }\end{array}$ \\
\hline 15 & Utilizo la fe para aliviar mis dolores & Utilizo mis creencias religiosas para aliviar mi dolor \\
\hline 16 & $\begin{array}{l}\text { Cuando tengo dolor intento hablar con alguien } \\
\text { y contarle lo que me pasa. Esto me ayuda a } \\
\text { soportarlo }\end{array}$ & $\begin{array}{l}\text { Cuando tengo dolor le cuento a alguien lo que me } \\
\text { pasa. Esto me ayuda a soportarlo }\end{array}$ \\
\hline 22 & $\begin{array}{l}\text { Hablo con la gente de mi dolor, porque el hablar } \\
\text { me ayuda a sentirme mejor }\end{array}$ & $\begin{array}{l}\text { Hablo de mi dolor porque me ayuda a sentirme } \\
\text { mejor }\end{array}$ \\
\hline
\end{tabular}

TABLA II

MATRIZ DE COMPONENTES Y VARIANZA TOTAL EXPLICADA EN LA ESCALA

\begin{tabular}{|c|c|c|c|}
\hline \multicolumn{4}{|c|}{ Autovalores iniciales } \\
\hline Componente & Total & $\%$ de la varianza & $\%$ acumulado \\
\hline 1 & 6495 & 27.062 & 27.062 \\
\hline 2 & 3405 & 14.189 & 41.251 \\
\hline 3 & 2505 & 10.436 & 51.686 \\
\hline 4 & 1726 & 7193 & 58.879 \\
\hline 5 & 1705 & 7106 & 65.986 \\
\hline 6 & 1430 & 5958 & 71.943 \\
\hline
\end{tabular}

asociada de 0,000. Datos que confirman la pertinencia de realizar un análisis factorial de la matriz de correlaciones y corroboran la validez del método de Componentes Principales para esta situación.

Los resultados sugieren la presencia de seis factores, que explican el $71 \%$ del acumulado. A consideración de los autores de la presente investigación, se decide mantener el nombre de los factores propuesto por los autores originales del cuestionario donde "Religión" hace referencia a la necesidad del uso de la religión o espiritualidad, así como al apoyo en la fe para hacer frente a la enfermedad para conseguir estabilidad y consuelo, "Catarsis" se trata de las verbali- zaciones que el paciente realiza sobre su dolor por el alivio que produce el hecho de comentar con otros el problema y buscar comprensión pero no tanto para buscar soluciones, "Distracción" describe el conjunto de acciones que el paciente lleva a cabo para desviar su atención sobre el dolor, "Autocontrol mental" se refiere a los esfuerzos cognitivos para intentar disminuir o controlar el dolor, "Autoafirmación" hace referencia a los ánimos que el paciente se da a sí mismo con el fin de no desfallecer y "Búsqueda de información" que alude a las consultas que realiza el individuo para saber más sobre su problema con el fin de poder controlar o solucionar su dolor. 
TABLA III

MATRIZ FACTORIAL DE COMPONENTES PRINCIPALES, ROTACIÓN VARIMAX. SE ELIMINARON LAS SATURACIONES INFERIORES A 0,50

\begin{tabular}{|c|c|c|c|c|c|c|}
\hline Ítems & Componente & & & & & \\
\hline & 1 & II & II & IV & V & VI \\
\hline 9 & 0,901 & & & & & \\
\hline 3 & 0,863 & & & & & \\
\hline 15 & 0,857 & & & & & \\
\hline 21 & 0,854 & & & & & \\
\hline 22 & & 0,936 & & & & \\
\hline 10 & & 0,899 & & & & \\
\hline 16 & & 0,809 & & & & \\
\hline 8 & & & 0,848 & & & \\
\hline 2 & & & 0,783 & & & \\
\hline 20 & & & 0,755 & & & \\
\hline 14 & & & 0,601 & & & \\
\hline 17 & & & & 0,828 & & \\
\hline 23 & & & & 0,817 & & \\
\hline 11 & & & & 0,761 & & \\
\hline 5 & & & & 0,745 & & \\
\hline 7 & & & & & 0,756 & \\
\hline 1 & & & & & 0,708 & \\
\hline 13 & & & & & 0,676 & \\
\hline 19 & & & & & 0,675 & \\
\hline 18 & & & & & & 0,755 \\
\hline 12 & & & & & & 0,682 \\
\hline 6 & & & & & & 0,662 \\
\hline 24 & & & & & & 0,521 \\
\hline & Distracción & $\begin{array}{l}\text { Búsqueda de } \\
\text { información }\end{array}$ & Religión & Catarsis & $\begin{array}{c}\text { Autocontrol } \\
\text { mental }\end{array}$ & Autoafirmación \\
\hline
\end{tabular}

\section{Evaluación de la fiabilidad}

La Tabla IV refleja los resultados alcanzados por el Coeficiente $-\alpha$ de Cronbach. De manera global se alcanzó una puntuación de 0,853, lo cual demuestra la naturaleza homogénea del test. Al eliminar un ítem se obtiene valores que oscilan entre 0,838 y 0,861, lo que indica que todos los ítems son pertinentes y contribuyen al carácter homogéneo del instrumento. Todas las escalas (factores] presentan índices de consistencia interna de moderados a altos, excepto la escala autoafirmación con 0,608.

La Tabla V muestra los resultados del análisis de la correlación entre los dos momentos de aplicación del instrumento, test y retest. Se puede apreciar que no existieron diferencias estadísticamente significativas, con valores que oscilan entre 0,793 y 0,879, lo que demuestra que el instrumento es confiable en relación con su estabilidad en el tiempo.

\section{DISCUSIÓN}

El auge en la evaluación de las estrategias de afrontamiento y el abordaje multidimensional del dolor ha dado lugar al desarrollo de múltiples instrumentos para evaluar las estrategias de afrontamiento en pacientes con dolor crónico (5-8). Sin embargo, la mayoría de estos instrumentos se han diseñado y validado en contextos culturales fuera de Cuba.

El cuestionario que se presenta emplea un lenguaje claro, sencillo de responder, con frases y términos que se ajustan al contexto social cubano, lo que suple en 
TABLA IV

RESULTADOS DE LA CONSISTENCIA INTERNA POR $\alpha$ DE CRONBACH GLOBAL, AL ELIMINAR UN ITEM Y POR FACTORES

\begin{tabular}{|c|c|c|}
\hline items & $\begin{array}{c}\alpha \text { al } \\
\text { eliminar } \\
\text { un ítem }\end{array}$ & $\begin{array}{c}\alpha \\
\text { por } \\
\text { Factores }\end{array}$ \\
\hline 9. Rezo para conseguir fuerza y guía sobre el problema & 0,842 & \multirow{4}{*}{$\begin{array}{c}\text { Factor I } \\
0,726\end{array}$} \\
\hline 3. Rezo para curar mi dolor & 0,844 & \\
\hline 15. Utilizo mis creencias religiosas para aliviar mi dolor & 0,842 & \\
\hline 21. Rezo para que mis dolores desaparezcan & 0,838 & \\
\hline 22. Hablo de mi dolor porque me ayuda a sentirme mejor & 0,846 & \multirow{3}{*}{$\begin{array}{c}\text { Factor } \| \\
0,851\end{array}$} \\
\hline $\begin{array}{l}\text { 10. Digo a los demás lo mucho que me duele, pues el compartir } \\
\text { mis sentimientos me hace sentir mejor }\end{array}$ & 0,846 & \\
\hline $\begin{array}{l}\text { 16. Cuando tengo dolor le cuento a alguien lo que me pasa. } \\
\text { Esto me ayuda a soportarlo }\end{array}$ & 0,841 & \\
\hline $\begin{array}{l}\text { 8. Hablo con alguien que pueda ayudarme a hacer frente } \\
\text { a mi dolor }\end{array}$ & 0,842 & \multirow{4}{*}{$\begin{array}{c}\text { Factor III } \\
0,939\end{array}$} \\
\hline 2. Cuando tengo dolor hablo con un profesional para que me ayude & 0,844 & \\
\hline 20. Busco algún amigo, familiar o profesional para que me aconseje & 0,843 & \\
\hline 14. Intento que me expliquen qué puedo hacer & 0,842 & \\
\hline 17. Cuando tengo dolor me concentro en su localización & 0,845 & \multirow{4}{*}{$\begin{array}{l}\text { Factor IV } \\
0,871\end{array}$} \\
\hline 23. Me concentro en el punto que más me duele & 0,847 & \\
\hline 11. Cuando tengo dolor me concentro en él & 0,849 & \\
\hline 5. Me olvido de todo y me concentro en mi dolor & 0,850 & \\
\hline 7. Cuando tengo dolor pienso en otra cosa & 0,858 & \multirow{4}{*}{$\begin{array}{c}\text { Factor V } \\
0,824\end{array}$} \\
\hline 1. Ignoro el dolor pensando en otra cosa & 0,861 & \\
\hline 13. Intento recrear mentalmente un paisaje & 0,847 & \\
\hline 19. Cuando tengo dolor imagino situaciones placenteras & 0,848 & \\
\hline 18. Me digo a mí mismo que tengo que ser fuerte & 0,845 & \multirow{5}{*}{$\begin{array}{c}\text { Factor V } \\
0,608\end{array}$} \\
\hline 12. Me doy ánimo para aguantar el dolor & 0,848 & \\
\hline 6. Cuando tengo dolor lucho para vencerlo & 0,856 & \\
\hline 24. Pienso que he de tener fuerza & 0,858 & \\
\hline 4. Busco algún amigo o allegado & 0,842 & \\
\hline$\alpha$ de Cronbach Global & 0,853 & \\
\hline
\end{tabular}


TABLA V

RESULTADO DE LA CORRELACIÓN TEST-RETEST Y $\alpha$ DE CRONBACH PARA LOS FACTORES

\begin{tabular}{|l|l|}
\hline \multicolumn{1}{|c|}{ Factores } & \multicolumn{1}{|c|}{$\begin{array}{c}\text { Correlación de } \\
\text { Pearson }\end{array}$} \\
\hline Distracción & $0,831^{* *}$ \\
\hline Búsqueda de información & $0,793^{* *}$ \\
\hline Religión & $0,814^{* *}$ \\
\hline Catarsis & $0,856^{* *}$ \\
\hline Autocontrol mental & $0,839^{* *}$ \\
\hline Autoafirmación & $0,879^{* *}$ \\
\hline
\end{tabular}

$\mathrm{p}<0,01$.

alguna medida la falta de instrumentos estandarizados en nuestro medio.

Constituye una herramienta metodológica y un instrumento de trabajo que permite a médicos, psicólogos, rehabilitadores y especialidades afines evaluar de manera fácil las estrategias de afrontamiento en pacientes con dolor crónico de la espalda en la población cienfueguera, patología que, pese a no existir reportes estadísticos o documentación en nuestro territorio, representa un importante problema de salud.

Con respecto al proceso de validación, el cuestionario presenta validez de contenido, lo que se alcanzó mediante el criterio de experto. Esto constituye un importante indicador de su valor y una justificación loable tenida en cuenta en el proceso de validación, pues esta alternativa de validez permite realizar una valoración crítica del constructo teórico del que se parte y las categorías o dimensiones que se proponen $(13,14)$.

Por otra parte, los resultados de la validez de construcción lógica confirmaron una buena adecuación muestral y fuertes correlaciones entre las variables, agrupándose los ítems en seis factores que explican el $71 \%$ del acumulado, lo que demuestra la medida que el instrumento reproduce la estructura original.

El análisis de componentes principales por el método de rotación con Kaiser mostró que existen reactivos 0 ítems más correlacionados entre sí, lo cual se expresa a través de la selección de seis componentes o factores.

Estos resultados coinciden a los obtenidos por Soriano y Monsalve en la validación original del instrumento y posteriormente en un análisis factorial confirmatorio del mismo $[8,15)$.

Estos autores obtuvieron un índice $\mathrm{KMO}$ de 0,861, con un test de esfericidad de Bartlett $(\chi 2=26367,928$; $\mathrm{gl}=276 ; \mathrm{p}=0,000) \mathrm{El}$ análisis paralelo indicó la retención de seis factores trabajando con el $95 \%$ sobre eigenvalues aleatorios; en este caso los seis factores explicaron el 68,37\% de la varianza. El factor 1 agrupó los ítems pertenecientes a "búsqueda de información", el factor 2 agrupó los ítems de "religión", el factor 3 los ítems de "autoafirmación", el factor 4 los pertenecientes a "autocontrol mental", el factor 5 los pertene- cientes a "catarsis" y el factor 6 los pertenecientes a "distracción" (15).

Otro aspecto relevante es la fiabilidad del cuestionario, a través de la cual se demostró la naturaleza homogénea del test.

El índice de Consistencia Interna en la versión original de Soriano y Monsalve al calcular el coeficiente $\alpha$ de Cronbach por dimensiones osciló en valores entre 0,74 y 0,94; resultados que están en consonancia con lo obtenidos en la presente investigación, con la diferencia que en este caso el $\alpha$ de Cronbach se determinó de manera global y al eliminar un ítem (8).

Asimismo, en recientes investigaciones realizadas por los autores originales del test sobre una muestra de 4389 pacientes con diagnóstico de dolor crónico, se obtuvieron resultados satisfactorios, donde todos los factores presentaron índices de consistencia interna de moderados a altos (15).

Con respecto a la estabilidad temporal del instrumento se obtienen resultados similares a los obtenidos en otras investigaciones, lo que confirma que el instrumento es confiable en relación con su estabilidad en el tiempo (15).

Es necesario afirmar que el instrumento que se propone refleja condiciones adecuadas y criterios válidos en la evaluación de las estrategias de afrontamiento en pacientes con diversos síndromes dolorosos, lo que ha quedado demostrado a partir de su amplia utilización en el terreno investigativo $(3,4,16)$.

A decir de sus autores originales, la aplicación de este instrumento de medida aportará, para investigaciones futuras, datos que permitan establecer el perfil adecuado del paciente frente al dolor crónico (8).

Podemos concluir diciendo que el Cuestionario Reducido de Afrontamiento al Dolor, adaptado desde una perspectiva lingüistica y cultural, reúne los requisitos de fiabilidad y validez necesarios para su uso en la población cienfueguera con dolor crónico de espalda.

\section{CONFLICTO DE INTERESES}

Los autores declaran no tener ningún conflicto de intereses.

\section{BIBLIOGRAFÍA}

1. Melzack R, Wall PD. Pain mechanisms: a new theory. Science. 1965;150(3699):971-9.

2. Bello Villanueva M, Benítez Lara M, Oviedo Trespalacios O. Características del dolor, aspectos psicológicos, calidad de vida y estrategias de afrontamiento en pacientes con dolor de espalda crónico en una ciudad de Colombia. Rev Colomb Anestesiol. 2017;45(4):310-6. DOI: 10.1016/j. rca.2017.07.002.

3. Amaya Ropero MC, Carrillo González GM. Apoyo social percibido y afrontamiento en personas con dolor crónico no maligno. Aquichan. 2015;15(4):461-74. DOI: 10.5294/ aqui.2015.15.4.2.

4. Soucase B, Monsalve V, Soriano JF. Afrontamiento del dolor crónico: el papel de las variables de valoración y estrategias de afrontamiento en la predicción de la ansiedad y la depresión en una muestra de pacientes con dolor crónico. Rev Soc Esp Dolor. 2005;12(1):8-16. 
5. Vitaliano P, Russo J, Carr J, Maiuro RD, Becker J. The Ways of Coping Checklist: revision and psychometric properties. Multivariate Behavior Research 1985;20(1):3-26. DOI: 10.1207/s15327906mbr2001_1.

6. Brown G, Nicassio P. Development of a questionnaire for the assessment of active and passive coping strategies in chronic pain patients. Pain 1987;31(1):53-64. DOI: 10.1016/0304-3959(87)90006-6.

7. Soriano J, Monsalve V. CAD: Cuestionario de Afrontamiento ante el Dolor Crónico. Rev Soc Esp Dolor. 2002;9(1):13-22.

8. Soriano J, Monsalve V. Validación del cuestionario de afrontamiento al dolor crónico reducido (CAD-R). Rev Soc Esp Dolor. 2004;11(7):27-34.

9. García JA, Valdés G, Martínez JC, Pedroso I. Epidemiología del dolor de espalda bajo. Invest Medicoquir. 2014;6(1):112-25.

10. Pomares Avalos A, Rodríguez Rodríguez T, Pomares Alfonso J. Variables psicológicas y clínicas presentes en pacientes portadores de dolor crónico de espalda. Revista Finlay. 2016;6(1):49-58.

11. Escobar Pérez J, Cuervo Martínez A. Validez de contenido y juicios de expertos: una aproximación a su utilización. Rev Avances en medición. 2008; 6[1]: 27-36.
12. Crespo Borges T. Dieciséis respuestas a preguntas sobre el criterio de expertos en investigaciones pedagógicas. Lima: Editorial San Marcos; 2007.

13. Galicia Alarcón LA, Balderrama Trápaga JA, Edel Navarro R. Validez de contenido por juicio de expertos: propuesta de una herramienta virtual. Apertura (Guadalajara, Jal.). 2017;9(2):42-53. DOI: 10.18381/ap.v9n2.993.

14. Urrutia Egaña M, Barrios Araya S, Gutiérrez Núñez M, Camus Pontificia MM. Métodos óptimos para determinar validez de contenido. Educación Médica Superior. 2014;28(3):547-58.

15. Soriano J, Monsalve V. CAD-R. Cuestionario de afrontamiento al dolor crónico: análisis factorial confirmatorio. ¿Hay diferencias individuales en sexo, edad y tipo de dolor? Rev Soc Esp Dolor. 2017;24(5):224-33. DOI: 10.20986/ resed.2017.3558/2016.

16. Peñarroya Baulíes A, Ballús-Creus C, Pérez Martínez J. Diferencias en el uso de las estrategias de afrontamiento, el catastrofismo y el estado de ánimo en función del tiempo de evolución del dolor crónico. Revista de la Sociedad Española del Dolor. 2011;18(2):77-83. 